\title{
Myositis and Liver Dysfunction due to Concomitant Therapy of Simvastatin and Itraconazole
}

\author{
Qiu-Ting Dong, Yuan-Lin Guo, Na-Qiong Wu, Yi-Da Tang, Li-Xin Jiang and Jian-Jun Li* \\ Division of Dyslipidemia, Cardiovascular Medicine, Fu Wai Hospital, Chinese Academy of Medical Sciences, Peking Union Medical College, China
}

\begin{abstract}
Simvastatin as an effective cholesterol-lowering medication is widely prescribed to treat cardiovascular diseases. Despite definite safety and efficacy, myopathy and liver dysfunction are the common adverse effects of simvastatin. Little information is available regarding the symptom starting and recovery period for a patient undergoing the side effect. We present here a 65-year-old male patient with both liver dysfunction and myositis due to the concomitant use of itraconazole $400 \mathrm{mg}$ and simvastatin $40 \mathrm{mg}$ daily.
\end{abstract}

Keywords: Myotoxicity; Simvastatin; Itraconazole

\section{Introduction}

Although statin, one kind of the hydroxymethylglutaryl coenzyme A (HMG-CoA) reductase inhibitor, have significant impact on lipid parameters and favorable safety profile in cardiovascular disease, especially in atherosclerotic disease, approximately 9-20\% statinstreated patients might develop myopathy which is commonly manifested by myalgias with or without plasma Creatine Kinase (CK) elevations [1-3]. High dose of statins, senility, genetic predisposition, female and co-administered with cytochrome P450 3A4 (CYP3A4) inhibitor are the main factors contributing to the adverse effect $[4,5]$. We here report a case with liver dysfunction and myopathy resulting from the co-administration of itraconazole $400 \mathrm{mg}$ daily and simvastatin $400 \mathrm{mg}$ daily. To our knowledge, one similar case has been previously published in the literature as a letter to the editor in another journal, whereas no data of time course were described in detail [6]. We presented this case for the purpose of adding to the subject and to alerting the practitioners to the interaction with simvastatin and itraconazole.

\section{Presentation of the Case}

A 65-year-old male patient with known coronary heart disease for 7 years, type 2 diabetes and primary hypertension for 4 years was referred because of simvastatin intolerant. The patient participated in the Heart Protection Study 2-Treatment of High-Density Lipoprotein (HDL) to Reduce the Incidence of Vascular Events (HPS2-THRIVE) Trial and randomized into the simvastatin $40 \mathrm{mg}$ arm in 2007. Receiving simvastatin $40 \mathrm{mg}$ daily for 4 years, the patient felt good and his liver function was normal on November $7^{\text {th }}, 2011$ (Table 1).

On November $13^{\text {th }}, 2011$, the patient consulted a dermatologist for tinea pedis, and was prescribed itraconazole $400 \mathrm{mg}$ daily for seven days. On December $1^{\text {st }}, 2011$ (ten days after termination of itraconazole), he felt right thigh ache, fatigue and weakness but can be tolerant.

On December $7^{\text {th }}$, the patient was routinely followed by the HPS2 investigator. Physical examination revealed that the myodynamia and muscular tension of his right thigh decreased. He denied having done any strenuous exercise, intramuscular injection, or recent trauma. The laboratory tests showed alanine aminotransferase (ALT) 208 IU/L (normal <40 IU/L), aspartate aminotransferase (AST) 264I U/L (normal $<40 \mathrm{IU} / \mathrm{L}$ ), creatine kinase (CK) $5704 \mathrm{IU} / \mathrm{L}$ (normal $<200$ IU/L), and CK-MB $224 \mathrm{IU} / \mathrm{L}$ (normal <24 IU/L) while above tests were normal one month earlier (Table 1). Urine tests were not abnormal. The doctor advised him to hospitalize immediately and stopped the simvastatin treatment.
On December $9^{\text {th }}$, the patient was admitted in hospital because reexamination laboratory tests showed ALT 194 IU/L, AST 246 IU/L, CK $3263 \mathrm{IU} / \mathrm{L}$ and CK-MB $1171 \mathrm{IU} / \mathrm{L}$. The routine blood tests, renal function tests and thyroid function tests were normal. His serum lipids profile demonstrated Triglyceride (TG) $0.82 \mathrm{mmol} / \mathrm{L}$, Total Cholesterol (TC) $3.08 \mathrm{mmol} / \mathrm{L}$, Low-Density Lipoprotein Cholesterol (LDL-C) $1.37 \mathrm{mmol} / \mathrm{L}$, High-Density Lipoprotein Cholesterol (HDL-C) $1.34 \mathrm{mmol} / \mathrm{L}$. He was diagnosed with liver dysfunction and myalgias and received therapies of intravenous hydration, polyene phosphatidylcholine and vitamin $\mathrm{C}$ intravenous drip.

On December $16^{\text {th }}$, the patient was discharged from the hospital. His serum levels of ALT, AST, CK, CK-MB and muscle symptoms had improved significantly (Table 1). The patient's medications included bay-aspirin, isosorbide mononitrate, atenolol, polyene phosphatidylcholine, vitamin C, glucurolactone and simvastatin $20 \mathrm{mg}$. At week 1 and week 3 after his discharge, we followed up him respectively, his serum ALT and CK both returned to normal levels (Table 1 and figure 1). We stopped the polyene phosphatidylcholine, vitamin $\mathrm{C}$ and glucurolactone therapy.

\begin{tabular}{|l|l|l|l|l|}
\hline Date & ALT (IU/L) & AST (IU/L) & CK (IU/L) & CK-MB (IU/L) \\
\hline $2011-11-07$ & 13.65 & 14.87 & Not done & Not done \\
\hline $2011-12-07$ & 208 & 264 & 5704 & 224 \\
\hline $2011-12-09$ & 194 & 246 & 3263 & 1171 \\
\hline $2011-12-10$ & 172 & 110 & 2014 & 39 \\
\hline $2011-12-12$ & 127 & 48 & 426 & 19 \\
\hline $2011-12-13$ & 87 & 31 & 212 & 14 \\
\hline $2011-12-14$ & 61 & 22 & 112 & 12 \\
\hline $2011-12-15$ & 54 & 21 & 96 & 8 \\
\hline $2011-12-23$ & 25 & Not done & 67.5 & Not done \\
\hline $2012-01-06$ & 15.6 & Not done & 76.5 & Not done \\
\hline
\end{tabular}

$\mathrm{ALT}=$ Alanine Aminotransferase; $\mathrm{AST}=$ Aspartate Aminotransferase; $\mathrm{CK}=$ Creatine Kinase; $\mathrm{CK}-\mathrm{MB}=$ Creatine Kinase-MB

Table 1: The results of the liver functions and laboratory tests.

*Corresponding author: Jian-Jun Li, MD, PhD, Division of Dyslipidemia, Cardiovascular Medicine, Fu Wai Hospital, Chinese Academy of Medical Sciences, Peking Union Medical College, Beijing 100037, P. R. China, E-mail: lijnjn@yahoo.com.cn

Received December 03, 2012; Accepted January 11, 2013; Published January 14,2013

Citation: Dong QT, Guo YL, Wu NQ, Tang YD, Jiang LX, et al. (2013) Myositis and Liver Dysfunction due to Concomitant Therapy of Simvastatin and Itraconazole. J Clin Case Rep 3: 247. doi:10.4172/2165-7920.1000247

Copyright: @ 2013 Dong QT, et al. This is an open-access article distributed under the terms of the Creative Commons Attribution License, which permits unrestricted use, distribution, and reproduction in any medium, provided the original author and source are credited. 


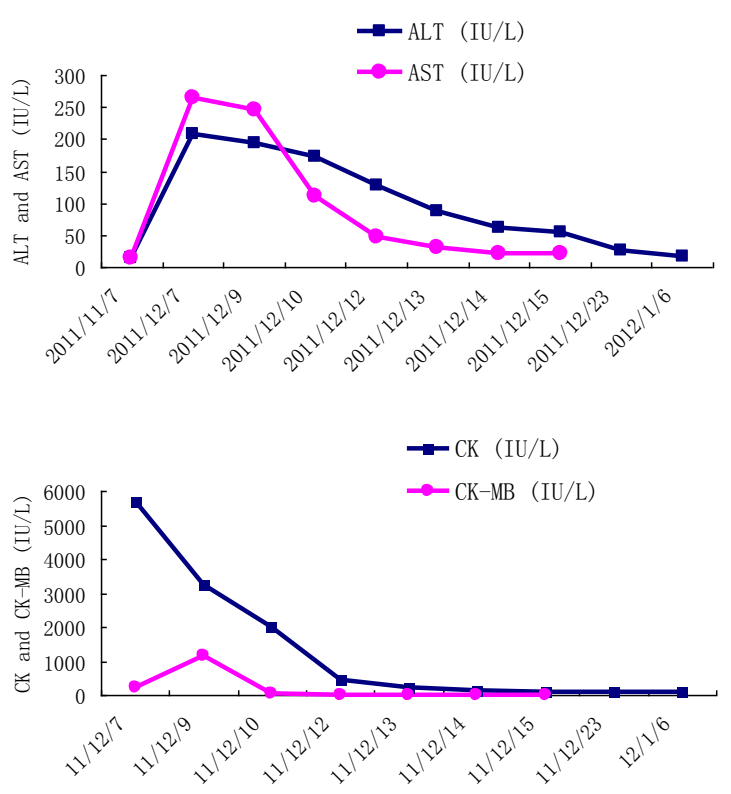

Figure 1: The time-dependent changes of serum test of alanine aminotransferase (ALT, figure 1a, blue line), aspartate aminotransferase (AST, figure $1 \mathrm{a}$, red line), creatine kinase (CK, figure $1 \mathrm{~b}$, red line) and CKMB (figure $1 \mathrm{~b}$, blue line).

\section{Discussion}

Primary and secondary prevention studies have been demonstrated that by lowering LDL-C levels, statin can significantly reduce morbidity and mortality in patients with coronary artery disease $[7,8]$. However, statin-related myotoxicity and hapotoxicity are major concerns in real world cardiovascular practice [9]. It has been demonstrated that HMG-CoA reductase inhibitors are primarily metabolized by the cytochrome P450 [10]. Of the several isoenzymes of the cytochrome P450, simvastatin is metabolized by CYP3A4 and can interfere the ubiquitin proteasome (coenzyme Q10 (CoQ10)) pathway which primarily maintains the architecture of skeletal muscle [5]. As a result, high concentration of simvastatin in serum will cause skeletal muscle damage. Additionally, the drugs that inhibit CYP3A4, such as antifungi drugs like itraconazole and nefazodone, will increase the plasma concentration of simvastatin dramatically and intensify the hazard of myopathy in patient treated with simvastatin especially at a high dose ( 40 or $80 \mathrm{mg}$ daily). Here we reported such case in detail whose myositis and liver dysfunction due to concomitant therapy of simvastatin and itraconazole, suggesting that doctors should be aware of the interaction between itraconazole and statins.

In fact, we searched the related reports and found only one case that described the adverse effect of simvastatin and itraconazole interaction [6]. This case was reported as a letter to the editor, which evinced a 67-year-old woman, who was treated with simvastatin $40 \mathrm{mg}$ daily for 2 years, suffered from myopathy 2 days later after combination with itraconazole $200 \mathrm{mg}$ daily for 5 days. The patient recovered after fluids and bicarbonate infusion for 5 days. Their case was a little similar to ours, especially, in the age of the patient (both elderly).

Our case was somehow different from the case mentioned above.

In our case, the patient started showing symptoms and laboratory abnormalities around 1 month after combination with itraconazole although the dose of itraconazole was $400 \mathrm{mg}$ daily. The possible causes might contain these factors such as age, gender, background disease, complications, genetic predisposition and drug combination [11]. Moreover, we present here a detailed time course regarding patient's data of laboratory tests. However, both cases showed that once myopathy or rhabdomyolysis was detected, withdrawal of statins were effective way to therapy [7]. While statins withdrawal will elevate vascular risks, thus the balance between statins' advantage and disadvantage should be struck [12]. We considered the high dose of simvastatin might play an important role leading to myopathy and liver dysfunction in this case. However, the patient might suffer from high risk of cardiovascular event if we discontinued simvastatin. So we reduced the dose of simvastatin to $20 \mathrm{mg}$ daily. However, in order to make clear of the definite reasons of myositis in this case, we should further determine the pharmacogenetic profile (e.g. polymorphisms of $3 \mathrm{~A} 4)$.

There is aboard consensus on aggressive cholesterol control in cardiovascular disease [13]. Agents such as simvastatin may be prescribed more frequently at higher dose accordingly. Practitioner should be more cautious in the interaction between anti-fungi agents such as itraconazole and simvastatin, especially in temporary treatment. It is much safer for patients needing anti-fungi drugs to reduce dose of primary statins or to change those statins which is not metabolized by CYP3A4.

\section{References}

1. Bruckert E, Hayem G, Dejager S, Yau C, Bégaud B (2005) Mild to moderate muscular symptoms with high-dosage statin therapy in hyperlipidemic patientsthe PRIMO study. Cardiovasc Drugs Ther 19: 403-414.

2. de Sauvage Nolting PR, Buirma RJ, Hutten BA, Kastelein JJ; Dutch ExPRESS Investigator Group (2002) Two-year efficacy and safety of simvastatin $80 \mathrm{mg}$ in familial hypercholesterolemia (the Examination of Probands and Relatives in Statin Studies With Familial Hypercholesterolemia [ExPRESS FH]). Am J Cardiol 90: 181-184.

3. Franc S, Dejager S, Bruckert E, Chauvenet M, Giral P, et al. (2003) A comprehensive description of muscle symptoms associated with lipid-lowering drugs. Cardiovasc Drugs Ther 17: 459-465.

4. Eckel RH (2010) Approach to the patient who is intolerant of statin therapy. $J$ Clin Endocrinol Metab 95: 2015-2022.

5. Grosshans BL, Ortiz D, Novick $P$ (2006) Rabs and their effectors: achieving specificity in membrane traffic. Proc Natl Acad Sci U S A 103: 11821-11827.

6. Saliba WR, Elias M (2005) Severe myopathy induced by the co-administration of simvastatin and itraconazole. Eur J Intern Med 16: 305.

7. Klopstock T (2008) Drug-induced myopathies. Curr Opin Neurol 21: 590-595.

8. Baigent C, Keech A, Kearney PM, Blackwell L, Buck G, et al. (2005) Efficacy and safety of cholesterol-lowering treatment: prospective meta-analysis of data from 90,056 participants in 14 randomised trials of statins. Lancet 366: 12671278.

9. Tiwari A, Bansal V, Chugh A, Mookhtiar K (2006) Statins and myotoxicity: a therapeutic limitation. Expert Opin Drug Saf 5: 651-666.

10. Igel M, Sudhop T, von Bergmann K (2001) Metabolism and drug interactions of 3-hydroxy-3-methylglutaryl coenzyme A-reductase inhibitors (statins). Eur $\mathrm{J}$ Clin Pharmacol 57: 357-364.

11. Vormfelde SV (2001) Safety of statins (hydroxymethyl glutaryl coenzyme a reductase inhibitors): different mechanisms of metabolism and drug transport may have clinical relevance. Arch Intern Med 161: 1012-1013.

12. Rodríguez-Yáñez M, Dávalos A, Castillo J (2008) Withdrawal from statins: implications for secondary stroke prevention and acute treatment. Int J Stroke 3: 85-87.

13. Gotto AM Jr, Moon JE (2012) Management of cardiovascular risk: the importance of meeting lipid targets. Am J Cardiol 110: 3A-14A. 